\title{
A NOTE ON 2-LOCAL REPRESENTATIONS OF C*-ALGEBRAS
}

\author{
Antonio M. Peralta
}

Abstract. We survey the results on linear local and 2-local homomorphisms and zero products preserving operators between $\mathrm{C}^{*}$-algebras, and we incorporate some new precise observations and results to prove that every bounded linear 2-local homomorphism between $\mathrm{C}^{*}$-algebras is a homomorphism. Consequently, every linear 2-local *-homomorphism between $\mathrm{C}^{*}$-algebras is a *-homomorphism.

Mathematics subject classification (2010): 46L05, 46L40.

Keywords and phrases: Local homomorphism, local * -homomorphism; 2-local homomorphism, 2local *-homomorphism; local representation; 2-local representation.

\section{REFERENCES}

[1] J. Alaminos, M. Bresar, J. Extremera, A. Villena, Characterizing homomorphisms and derivations on $C^{*}$-algebras, Proc. Roy. Soc. Edinb. A 137, 1-7 (2007).

[2] C. BADEA, The Gleason-Kahane-Zelazko theorem and Gel'fand theory without multiplication, Université de Paris-sud, Département de mathématiques, Orsay, France, 1993.

[3] C. Batty, L. MolnaR, On topological reflexivity of the groups of *-automorphisms and surjective isometries of $B(H)$, Arch. Math. 67 (1996), 415-421.

[4] M. BRESAR, Characterization of derivations on some normed algebras with involution, J. Algebra 152 (1992), 454-462.

[5] M. BREŠAR, P. SŠEMRL, On local automorphisms and mappings that preserve idempotents, Studia Math. 113, 2 (1995), 101-108.

[6] M. Burgos, F. J. Fernández-Polo, J. J. Garcés, J. Martínez Moreno, A. M. Peralta, Orthogonality preservers in $C^{*}$-algebras, $J B^{*}$-algebras and $J B^{*}$-triples, J. Math. Anal. Appl. 348 (2008), 220-233.

[7] M. Burgos, F. J. Fernández-Polo, J. J. Garcés, A. M. Peralta, Orthogonality preservers Revisited, Asian-European Journal of Mathematics 2, 3 (2009), 387-405.

[8] M. Burgos, F. J. Fernández-Polo, J. J. GArCÉs, A. M. Peralta, Local triple derivations on $C^{*}$-algebras, Communications in Algebra 42 (2014), 1276-1286.

[9] M. Burgos, F. J. Fernández-Polo, A. M. Peralta, Local triple derivations on $C^{*}$-algebras and $J^{*}{ }^{*}$-triples, Bull. London Math. Soc. 46 (2014) 709-724. doi:10.1112/blms/bdu024

[10] E. CARTAn, Sur les domaines bornés homogenes de l'esplace de $n$ variables complexes, Abh. Math. Sem. Univ. Hamburg 11 (1935), 116-162.

[11] CH.-H. Chu, Jordan Structures in Geometry and Analysis, Cambridge Tracts in Math. 190, Cambridge. Univ. Press, Cambridge, 2012.

[12] R. CRIST, Local automorphisms, Proc. Amer. Math. Soc. 128 (1999), 1409-1414.

[13] H. G. DALES, Banach algebras and automatic continuity, London Mathematical Society Monographs (New Series), Volume 24, Oxford Science Publications. The Clarendon Press, Oxford University Press, New York, 2000.

[14] F. J. Fernández-Polo, A. Molino Salas, A. M. Peralta, Local triple derivations on real $C^{*}$-algebras and $J^{*}$-triples, to appear in Bull. Malaysian Math. Sci. Soc.

[15] A. FošNER, 2-local Jordan automorphisms on operator algebras, Studia Math. 209, 3 (2012), 235246.

[16] J. J. GARCÉs, A. M. PERALTA, Orthogonal forms and orthogonality preservers on real function algebras, Linear and Multilinear Algebra, 62, 3 (2014), 275-296. DOI:10.1080/03081087.2013.772998. 
[17] S. R. Garcia, J. Tener, Unitary equivalence of a matrix to its transpose, J. Operator Theory 68, 1 (2012), 179-203.

[18] J. J. Garcés, A. M. Peralta, D. Puglisi, M. I. Ramírez, Orthogonally additive, orthogonality preserving, holomorphic mappings between $C^{*}$-algebras, Abstr. Appl. Anal. 2013, Art. ID 415354, 9 pp.

[19] A. M. Gleason, A characterization of maximal ideals, J. Analyse Math. 19 (1967), 171-172.

[20] U. HAAgERUP, The Grothendieck inequality for bilinear forms on $C^{*}$-algebras, Adv. Math. 56 (1985), 93-116.

[21] D. Hadwin, J. Li, Local derivations and local automorphisms, J. Math. Anal. Appl. 290, 2 (2004), 702-714.

[22] P. R. Halmos, A Linear Algebra Problem Book, Dolciani Math. Exp., vol. 16, Math. Assoc. America, Washington, DC 1995.

[23] B. E. Johnson, Local derivations on $C^{*}$-algebras are derivations, Trans. Amer. Math. Soc. 353 (2001), 313-325.

[24] R. V. KADISOn, Local derivations, J. Algebra 130 (1990), 494-509.

[25] J. P. KAHANE, W. ŻELAZKO, A characterization of maximal ideals in commutative Banach algebras, Studia Math. 29 (1968), 339-343.

[26] W. KAUP, A Riemann mapping theorem for bounded symmetric domains in complex Banach spaces, Math. Z. 183 (1983), 503-529.

[27] S. O. Kim, J. S. Kim, Local automorphisms and derivations on $\mathbb{M}_{n}$, Proc. Amer. Math. Soc. 132, 5 (2004), 1389-1392.

[28] S. O. Kim, J. S. Kim, Local automorphisms and derivations on certain $C^{*}$-algebras, Proc. Amer. Math. Soc. 133, 11 (2005), 3303-3307.

[29] S. KowAls KI, Z. SLODKOWS KI, A characterization of multiplicative linear functionals in Banach algebras, Studia Math. 67 (1980), 215-223.

[30] D. R. Larson And A. R. Sourour, Local derivations and local automorphisms of $B(X)$, Proc. Sympos. Pure Math. 51, Part 2, Providence, Rhode Island 1990, pp. 187-194.

[31] J. LI, Z. PAN, Annihilator-preserving maps, multipliers, and derivations, Linear Algebra Appl. 423 (2010), 5-13.

[32] J.-H. LiU, N.-C. Wong, 2-Local automorphisms of operator algebras, J. Math. Anal. Appl. 321 (2006), 741-750.

[33] M. MACKeY, Local derivations on Jordan triples, Bull. London Math. Soc. 45, 4 (2013), 811-824. doi: $10.1112 / \mathrm{blms} / \mathrm{bdt} 007$

[34] L. Molnar, 2-local isometries of some operator algebras, Proc. Edinburgh Math. Soc. 45 (2002), 349-352.

[35] L. MolnaR, Local automorphisms of operator algebras on Banach spaces, Proc. Amer. Math. Soc. 131 (2003), 1867-1874.

[36] L. MolnaR, Selected preserver problems on algebraic structures of linear operators and on function spaces, Lecture Notes in Mathematics, 1895. Springer-Verlag, Berlin, 2007.

[37] L. MOLNAR AND P. ŠEMRL, Local automorphisms of the unitary group and the general linear group on a Hilbert space, Expo. Math. 18 (2000), 231-238.

[38] F. D. Murnaghan, On the unitary invariants of a square matrix, An. Acad. Brasil. Cienc. 26 (1954), $1-7$.

[39] C. Pearcy, A complete set of unitary invariants for $3 \times 3$ complex matrices, Trans. Amer. Math. Soc. 104 (1962), 425-429.

[40] G. K. Pedersen, $C^{*}$-algebras and their automorphism groups, Academic Press, London 1979.

[41] F. Pop, On local representation of von Neumann algebras, Proc. Amer. Math. Soc. 132, 12 (2004), 3569-3576.

[42] S. SAKAI, $C^{*}$-algebras and $W^{*}$-algebras, Springer-Verlag, Berlin 1971.

[43] P. ŠEMrL, Local automorphisms and derivations on B(H), Proc. Amer. Math. Soc. 125 (1997), 26772680.

[44] V. Shul'man, Operators preserving ideals in $C^{*}$-algebras, Studia Math. 109 (1994), 67-72.

[45] W. Specht, Zur Theorie der Matrizen. II., Jber. Deutsch. Math. Verein. 50 (1940), 19-23.

[46] H. UpmeIER, Symmetric Banach Manifolds and Jordan $C^{*}$-algebras, North Holland, Amsterdam, 1985. 
[47] A. R. Villena, Automatic continuity in associative and nonassociative context, Irish Math. Soc. Bull. 46 (2001), 43-76.

[48] NG.-CH. Wong, Zero product preservers of $C^{*}$-algebras, Contemp. Math. 435 (2007), 377-380.

[49] W. ŻELAZKO, A characterization of multiplicative linear functionals in complex Banach algebras, Studia Math. 30 (1968), 83-85.

[50] J. ZHANG, F. PAN, A. YANG, Local derivations on certain CSL algebras, Linear Algebra Appl. 413 (2006), 93-99. 